\title{
Ant-Colony Based Optimal MC-CDMA Multiuser Detector
}

\author{
Samer L. Hijazi \\ Dept. of Electrical \\ and Computer Engineering \\ Kansas State University \\ Manhattan, KS 66506-5204 \\ Email: hijazi@ksu.edu
}

\author{
Andrew J. Best \\ Dept. of Electrical \\ and Computer Engineering \\ Kansas State University \\ Manhattan, KS 66506-5204 \\ Email: ajb1066@ksu.edu
}

\author{
Balasubramaniam Natarajan \\ Dept. of Electrical \\ and Computer Engineering \\ Kansas State University \\ Manhattan, KS 66506-5204 \\ Email: bala@ksu.edu
}

\author{
Sanjoy Das \\ Dept. of Electrical \\ and Computer Engineering \\ Kansas State University \\ Manhattan, KS 66506-5204 \\ Email: sdas@ksu.edu
}

\begin{abstract}
In this paper, we present a novel multiuser detection (MUD) technique based on ant colony optimization (ACO), for synchronous multi-carrier code division multiple access (MCCDMA) systems. ACO algorithms are based on the cooperative foraging strategy of real ants. While an optimal MUD design using an exhaustive search method is prohibitively complex, we show that the ACO based MUD converges to the optimal MUD BER performance in relatively few iterations providing $98 \%$ saving in computational complexity.
\end{abstract}

Index multi-user detection (ML-MUD), ant colony optimization

\section{INTRODUCTION}

Multi-carrier code division multiple access (MC-CDMA)[1] has emerged as a powerful alternative to conventional direct sequence CDMA (DS-CDMA)[2] in mobile wireless communications. In MC-CDMA, each user's data symbol is transmitted simultaneously over $N$ narrowband subcarriers, with each subcarrier encoded with one chip of a preassigned spreading code. Multiple users are assigned unique, orthogonal (or pseudo-orthogonal) code. That is, while DSCDMA spreads in the time domain, MC-CDMA applies the same spreading sequences in the frequency domain. While the performance of MC and DS-CDMA is identical in an additive white gaussian noise (AWGN) channel, MC-CDMA has been shown to outperform DS-CDMA in multipath channels [1], [3].

In an uplink of a CDMA based system, different users' signals experience independent random amplitude and phase distortions, resulting in a loss of orthogonality among users at the base station. This in turn results in multi-user interference (MUI) which limits the capacity as well as performance of the CDMA system. While single-user receivers are optimal receivers for a CDMA system with orthogonal user signals, they are not optimal in the presence of MUI. Therefore, multiuser detection (MUD) techniques were proposed by Verdu [4] in order to effectively combat MUI in DS-CDMA systems. Multiuser detectors (MUD) jointly demodulate all users' symbols and have been proved to be the optimal reception technique for DS-CDMA systems in fading channels [4].

Optimal and sub-optimal MUDs have also been proposed for MC-CDMA systems and have been a focus of research in recent years [5],[6]. The maximum likelihood (ML) MUD offers the best bit error rate (BER) performance among all multi-user detectors (and is called the optimal MUD receiver). The ML-MUD maximizes the joint probability by evaluating a maximum-likelihood function over the set of all possible users' symbol sequences forming an NP-complete optimization problem. Thus, the optimal MUD has a computational complexity that increases exponentially with the number of users and, hence, is impractical to implement. To overcome this limitation, several suboptimal techniques have been considered [6].

Since optimal MUD design can be modelled as an NPcomplete optimization problem, many techniques used to solve NP-complete problems can be applied to optimal receiver design. One such approach involves the use of nature-inspired optimization techniques. Over the past few decades, there have been numerous optimization algorithms developed based on theories of evolution and swarm intelligence. These include, evolutionary algorithms such as the genetic algorithm [7], evolutionary programming [8], particle swarm optimization [9], and ant-colony optimization (ACO)[10].

In this paper, we propose a novel low complexity optimal MUD for synchronous MC-CDMA uplink based on ACO. To the best of the authors' knowledge, this is the first ever attempt in implementing an optimal MUD for MC-CDMA using particle swarm intelligence. Ant Colony Optimization is based on the foraging strategy of real ants. In the ACO approach, several artificial ants perform a sequence of operations iteratively. In each iteration, ants are guided by a problem-specific greedy heuristic that aid their search for good solutions. In addition, ants seek solutions using information gathered previously to perform their search in the vicinity of good solutions. A strong reason for choosing the ACO approach is that it has been shown to outperform genetic algorithm based approaches for some NP-complete problems (e.g., travelling salesman problem [11]). In fact, in [12], the authors demonstrate that an ACO based MUD for DSCDMA provides optimal bit-error-rate performance with a lower computational complexity than a GA-based MUD. In this paper, simulation results demonstrate that our ACO based MUD for MC-CDMA is able to achieve the optimal BER bound with $98 \%$ lower complexity relative to an exhaustive search method.

This paper is organized as follows. In Section II, we provide the MC-CDMA system model and set-up the optimal MUD optimization problem in a synchronous up-link. In Section IV, we introduce ACO and present our novel ACO based MUD algorithm. In Section V, we present our simulation parameters, results, and an evaluation of the new algorithm. Finally, in 
Section VI, conclusions and future work are presented.

\section{MC-CDMA SYSTEM MODEL}

In this paper, we consider a synchronous uplink MC-CDMA system with $N$ carriers and $K$ users where each user is assigned a unique spreading code $\underline{\beta}^{k}=\left[\beta_{1}^{k}, \beta_{2}^{k}, \ldots, \beta_{N}^{k}\right]^{T}$. Figure 1 illustrates the $k^{\text {th }}$ transmitter and receiver model. The input to the IFFT block in Figure 1(a) corresponds to

$$
\underline{s}^{k}=\underline{\beta}^{k} \cdot b^{k} .
$$

Here, $\underline{s}^{k}$ is a $N \times 1$ vector whose elements are the $k^{t h}$ user's transmitted components on each carrier, and $b^{k}$ is the data symbol ( \pm 1 for BPSK ) of the $k^{t h}$ user.

\section{A. Channel Model}

In this work, we assume a slowly varying multipath channel for all users in the system. Multipath propagation in time translates into frequency selectivity in the frequency domain. While there is frequency selectivity over the entire bandwidth, each subcarrier experiences a flat fade. This is because $\Delta f<<(\Delta f)_{c}<B W$ (where $(\Delta f)$ is the spacing between carriers, $(\Delta f)_{c}$ is the coherence bandwidth of the channel and $B W$ represents the total transmission bandwidth). Since we assume an uplink, each user has an independent set of fading parameters - the $i^{t h}$ subcarrier for each user experiences a Rayleigh-distributed attenuation, $\alpha_{i}^{k}$, and a phase offset, $\phi_{i}^{k}$. The Rayleigh fades for each user are correlated across subcarriers with the correlation between channel fades $\alpha_{i}^{k}$ and $\alpha_{j}^{k}$ corresponding to [13] [14]

$$
\rho_{i, j}=\frac{1}{1+\left(\frac{\Delta f_{i, j}}{\left(\Delta f_{c}\right)}\right)^{2}}
$$

where $\Delta f_{i, j}$ is the frequency separation between subcarriers $i$ and $j$. Also, we assume that we have $L$ fold frequency diversity where $L$ is defined as the ratio between the total bandwidth and the coherence bandwidth.

The net effect of the frequency selective channel on $k^{t h}$ user's signal can be modelled as

$$
\underline{h}^{k}=\left[\alpha_{1}^{k} e^{j \phi_{1}^{k}}, \alpha_{2}^{k} e^{j \phi_{2}^{k}}, \cdots, \alpha_{N}^{k} e^{j \phi_{N}^{k}}\right] .
$$

\section{B. MC-CDMA Receiver}

Assuming the MC-CDMA signal is transmitted through a slowly varying frequency selective fading channel, the $k^{\text {th }}$ user received signal vector at the output of the FFT (in Figure 1(b)) block can be represented as

$$
\begin{aligned}
\underline{r}^{k} & =\underline{h}^{k} \odot \underline{s}^{k}+\underline{n}^{k} \\
& =\underline{h}^{k} \odot \underline{\beta}^{k} b^{k}+\underline{n}^{k}
\end{aligned}
$$

where, $\underline{r}^{k}$ is a vector of dimension $N \times 1$; the operator $(\odot)$ represents an element wise multiplication of two vectors, and $\underline{n}^{k}$ is a $N \times 1$ vector of additive white gaussian noise samples. Assuming perfect phase synchronization (i.e., the channel phases are traced and removed perfectly at the receiver), the received vector can be redefined as

$$
\underline{r}^{k}=\underline{C}_{c h}^{k} b^{k}+\underline{n}^{k} .
$$

Here, $\underline{C}_{c h}^{k}=\left[\alpha_{1}^{k} \beta_{1}^{k}, \cdots, \alpha_{N}^{k} \beta_{N}^{k}\right]^{T}$. Consider all users received signal simultaneously, the output of the FFT block at the base station can be represented as

$$
\underline{r}=\sum_{k=1}^{K} \underline{r}^{k}=\mathbf{C}_{\mathbf{C H}} \underline{b}+\underline{n}
$$

where $\underline{b}$ is the vector of users' data defined as $\left[b^{1}, b^{2}, \ldots, b^{K}\right]^{T} ; \underline{n}$ is a vector of independent additive white Gaussian noise (AWGN) samples on each carrier, and

$$
\mathbf{C}_{\mathbf{C H}}=\left[\underline{C}_{c h}^{1} \cdots \underline{C}_{c h}^{K}\right] .
$$

\section{OPTIMAL MC-CDMA MUD}

The optimal MUD simultaneously detects all users' data to jointly minimize the effects of MUI. The optimal MUD is the maximum likelihood receiver that yields the optimal estimate of the transmitted data, $\underline{\hat{b}}$.

$$
\begin{aligned}
\underline{\hat{b}} & =\underset{\underline{\hat{b}}}{\operatorname{argmax}}\{P(\underline{\hat{b}}=\underline{b} \mid \underline{r})\} \\
& =\underset{\underline{\hat{b}}}{\operatorname{argmax}}\left\{P\left(\underline{r}=\mathbf{C}_{\mathbf{C H}} \underline{\hat{b}}+\underline{n} \mid \underline{b}\right)\right\} \\
& =\underset{\underline{\hat{b}}}{\operatorname{argmax}}\left\{P\left(\underline{n}=\underline{r}-\mathbf{C}_{\mathbf{C H}} \underline{\hat{b}} \mid \underline{b}\right)\right\}
\end{aligned}
$$

The joint pdf of the noise corresponds to

$$
p(\underline{n})=\frac{1}{(2 \pi)^{N / 2} \sigma} e^{-\frac{1}{2 \sigma^{2}} \underline{n}^{H}|\mathbf{I}|^{-1} \underline{n}}
$$

where $\sigma^{2}$ is the variance of the noise and $N$ is the number of carriers. Combining Eqns. (10) and (11)

$$
\begin{aligned}
\underline{\hat{b}}= & \underset{\underline{\hat{b}}}{\operatorname{argmin}}\left\{\underline{n}^{H}|\mathbf{I}|^{-1} \underline{n}\right\} \\
= & \underset{\underline{\hat{b}}}{\operatorname{argmin}}\left\{\underline{n}^{H} \underline{n}\right\} \\
= & \left.\underset{\underline{\hat{b}}}{\operatorname{argmin}}\left\{\left(\underline{r}-\mathbf{C}_{\mathbf{C H}} \underline{\hat{b}}\right)^{H}\left(\underline{r}-\mathbf{C}_{\mathbf{C H}} \underline{\hat{b}}\right)\right\}\right) \\
= & \underset{\underline{\underline{b}}}{\operatorname{argmin}}\left\{\underline{r}^{H} \underline{r}-\underline{\hat{b}}^{H} \mathbf{C}_{\mathbf{C H}}{ }^{H} \underline{r}-\underline{r}^{H} \mathbf{C}_{\mathbf{C H}} \underline{\hat{b}}\right. \\
& \left.+\underline{\hat{b}}^{H} \mathbf{C}_{\mathbf{C H}}{ }^{H} \mathbf{C}_{\mathbf{C H}} \underline{\hat{b}}\right\}
\end{aligned}
$$

Ignoring all terms that are independent of $\underline{\hat{b}}$, the optimal MUD for MC-CDMA systems corresponds to

$$
\begin{aligned}
\underline{\hat{b}}= & \underset{\underline{\hat{b}}}{\operatorname{argmax}}\left\{Q(\underline{\hat{b}})=2 \Re \mathrm{e}\left\{\underline{\hat{b}}{ }^{H} \mathbf{C}_{\mathbf{C H}}{ }^{H} \underline{r}\right\}\right. \\
& \left.-\underline{\hat{b}}^{H} \mathbf{C}_{\mathbf{C H}}{ }^{H} \mathbf{C}_{\mathbf{C H}} \underline{\hat{b}}\right\}
\end{aligned}
$$

Inspecting Eqn. (13), we observe that the optimal MUD consists of a difference of two terms. Only the first term depends on the received signal vector. However, it has been premultiplied by $\mathbf{C}_{\mathbf{C H}}{ }^{H}$ and the product is nothing but the output of the maximum ratio combining receiver (MRC). Hence, MRC outputs represent sufficient statistics to perform maximum likelihood detection. Furthermore, it can be seen that MRC output provides the optimal estimation of the transmitted data symbol if a single user is considered. Because MRC receivers are simple to implement and provide optimal performance for one user, they are often implemented in systems with multiple users. Since the MRC receiver does not jointly minimize the affects of MUI from other users, it is suboptimal and considered a single user receiver.

Similar to optimal DS-CDMA MUD [4], the solution for the optimal MC-CDMA MUD requires an exhaustive search over 
a set of $M^{K}$ possible solution vectors where $M$ is the number of points in the signal constellation (e.g., $M=2$ for BPSK) and $K$ is the number of users. The complexity of this receiver increases exponentially with the number of users. Therefore, it is impractical to implement.

By noting the similarities between the optimal DS-CDMA MUD and the optimal MC-CDMA MUD, it can be easily shown that the optimal MC-CDMA MUD problem belongs to a large class of combinatorial problems known as NPcomplete optimization problems. NP-complete problems are optimization problems (e.g., the traveling salesman and integer programming problems) that cannot be solved in polynomial time and the best solution technique is to implement an exhaustive search over all possible solutions. Therefore, in order to solve an NP-complete problem for any non-trivial problem size, one of the following approaches is used: (1) Approximation: An algorithm which quickly finds a suboptimal solution which is within a certain range of the optimal one; (2) Probabilistic: An algorithm which provably yields good average runtime behavior for a given distribution of the problem instances; and (3) Heuristic: An algorithm which works "reasonably well" on many cases, but for which there is no proof that it is always quickly yields a good solution (e.g., evolutionary techniques).

In recent years, particle swarm intelligence has inspired optimization algorithms that have been proposed for NPcomplete optimization problems. Ant colony optimization (ACO) is one such technique that is discussed in the following section. In [11], Dorigo showed that ACO is well suited in solving NP-complete problems (specifically, the traveling salesman problem). In this paper, we introduce ACO to the optimal MUD to design a realizable MC-CDMA optimal MUD receiver.

\section{Ant Colony Optimization for MC-CDMA SYSTEMS}

ACO is an attractive technique that is very effective in solving optimization problems that have discrete and finite search space. Since the optimal MUD design problem involves a search process across finite number of possible solutions, ACO is an ideal candidate to solve this problem.

\section{A. Ant Colony Optimization (ACO)}

ACO is based on the behavior of a colony of ants searching for food. In the ACO approach, several artificial ants perform a sequence of operations iteratively as shown in Figure 2.

To find a solution employing ACO, several iterations of artificial ants follows the flowchart shown in Figure 2. Within each iteration, several ants search in parallel for good solutions in the solution space. In each iteration of the algorithm, one or more ants are allowed to execute a move, leaving behind a pheromone trail for others to follow. An ant traces out a single path, probabilistically selecting only one element at a time, until an entire solution vector is obtained. In the following iterations, the traversal of ants is guided by the pheromone trails, i.e., the stronger the pheromone concentration along any path, the more likely an ant is to include that path in defining a solution. In each iteration, the quality of produced solution is estimated via a cost function. This estimate of solution quality is essential in determining whether or not to deposit pheromones on the traversal path. In addition to the pheromone values, the ants are also guided by a problem-specific greedy heuristic (desirability function) to aid in its search for good solutions.

It is easy to see that, as the search progresses, deposited pheromone dominates ants' selectivity, reducing the randomness of the algorithm. Therefore, ACO is an exploitive algorithm. It seeks solutions using information gathered previously, and performs its search in the vicinity of good solutions. However, since the ant's movements are stochastic, ACO is also an exploratory algorithm that samples a wide range of solutions in the solution space. This exploratory-exploitive approach is characteristic of many heuristic based optimization approaches, including GAs, taboo search and particle swarm. We can easily extend this general optimization technique to our MC-CDMA MUD problem as detailed in the next subsection.

\section{B. ACO based MUD}

The first stage in designing our ACO-based MUD involves the selection of ACO parameters that fit the optimization problem in Eqn.(13). In the MC-CDMA MUD problem, the solution corresponds to $\underline{b}_{o p t}$ which is a vector of length $K$. Each element of the solution vector takes one out of $M$ possible values, where $M$ is the constellation size. In this paper, we assume BPSK modulation, i.e., $M=2$. Therefore, $M^{K}$ possible solutions exist ( $2^{K}$ for BPSK). In our ACO algorithm, every ant builds a solution vector in each iteration. This building process is accomplished via $K$ jumps inside a $2 \times K$ table. The first row in this table represents an initial solution. The second row is merely the complement of the first row. Thus, any solution (out of the $2^{K}$ possible solutions) can be formed by selecting $K$ elements from this table, one element from each column. Hence, in each jump, the ant selects (based on a desirability function and pheromone concentration) either the initial solution element or its complement. When employing higher order modulation schemes, the dimensions of solution table becomes $M \times K$ with the first row containing the initial solution and each column containing one of the remaining $M-1$ possible data symbols. Similar to the presented case which employs BPSK, the solution is formed by selecting a set of $K$ elements, one from each column.

In single-user receivers, a suboptimal solution vector $\left(\underline{b}_{s u}\right)$ is created by performing hard decisions based on single user receiver outputs. In this paper, we employ the output of the MRC based single user receiver as the initial solution vector (i.e., $\left.\underline{b}_{s u}=\underset{\underline{b}_{s u} \in\{ \pm 1\}}{\operatorname{argmax}}\left\{\mathbf{C}_{\mathbf{C H}} \underline{r}\right\}\right)$. In the ACO algorithm, all ants begin their search at a specific position along the $\left(\underline{b}_{s u}\right)$ vector. The ants cyclically move down the $\underline{b}_{s u}$ vector, selecting the best element at each stage. The value of the element chosen by an ant is derived from the corresponding element values in either $\underline{b}_{s u}$ or $\underline{\bar{b}}_{s u}\left(\underline{\bar{b}}_{s u}=\left[\bar{b}_{s u}^{1} \bar{b}_{s u}^{2} \cdots \bar{b}_{s u}^{K}\right]^{T}\right.$ where $\bar{b}_{s u}^{l}=+1$ if $b_{s u}^{l}=-1$ and vice-versa $\forall l$ ). The desirability function is used to help the ant decide if a particular element value of the solution vector should come from $\underline{b}_{s u}$ instead of $\underline{b}_{s u}$. Since the magnitude of the conventional single user receiver outputs provide a rough estimate of the quality of users' hard decisions, it is used in evaluating the desirability function of the ants. The desirability function for an ant starting at $j^{t h}$ 
element in the $\underline{b}_{s u}$ vector is defined as:

$$
D(j)=\frac{1}{2+\left|R^{(j)}\right|}
$$

where $R^{(j)}=\underline{C}_{c h}^{(j)} \underline{r}$ is the soft decision value of the $j^{t h}$ received data symbol. Eqn.(14) reflects the fact that when $R^{(j)}=0, b_{s u}^{(j)}$ and $\bar{b}_{s u}^{(j)}$ are equally likely to be chosen. As the ant moves along the elements of the solution vector, the desirability function at the $(j+i)^{t h}$ stage can be redefined as follows:

$$
D(j+i)=\frac{1}{2+\left|R^{(j+i)}\right|+\sum_{l \in C}\left|R^{(l)}\right|} .
$$

where $C$ is a set of positions where the ant had previously selected $\bar{b}_{s u}$ element values. The desirability function defined in Eqns.(14) and (15) ensure that an ant does not significantly deviate from the initial solution. For example, if an ant chooses the element value from $\underline{\underline{b}}_{s u}$ at the $j^{\text {th }}$ position, its desirability to select another element value from $\underline{b}_{s u}$ decreases. Therefore, ants' starting positions in a single iteration should be as far as possible from one another along the solution vector. It is also important to note that while restricting ants' movements to the vicinity of the initial solution is not a necessary operation, but it is useful when the reliability of initial data estimates is high.

The second challenge in designing ACO based MUD algorithm, is to develop a meaningful pheromone deposition mechanism. In our algorithm, pheromones are deposited in a $2 \times K$ table where the first row corresponds to the elements of $\underline{b}_{s u}$, and the second row corresponds to the elements of $\underline{b}_{s u}$. At the beginning of the search process, the pheromone table has equal amounts (unity) of pheromones in all of its entries. As the search progresses, pheromones are deposited and evaporated based on the path traversed by the ants. The deposition rate $(D R)$ and evaporation rate $(E R)$ are parameters of the ACO. In our algorithm, the $D R$ and $E R$ are inversely related to the number of iterations, $V$. At any stage during the search, the higher the pheromone value in an entry (in the pheromone table), the probability of selecting the corresponding element value from $\underline{b}_{s u}$ or $\underline{\bar{b}}_{s u}$ is greater. Since $Q(\underline{b})$ determines the quality of a solution, it is used to control the amount of pheromone deposition. Furthermore, we use the elitism philosophy in our pheromone deposition mechanism, i.e., only the ants that find good paths ("elite ant") are allowed to deposit pheromones. Furthermore, if ants find excessively poor solutions ("weak ant"), pheromones are removed from those paths.

Our complete ACO based MUD algorithm is summarized below:

- Create a $2 \times K$ pheromone table, $P T \in \Re^{2 \times K}$; $P T(m, n)=1 \forall m, n$ [pheromone values are initialized]

- Set $\underline{b}_{\text {elite }}=\underline{b}_{s u}$ where $\underline{b}_{\text {elite }}$ is the best solution found.

- for iteration $=1: V,\{$

1) Decide the starting positions for $A$ ants $\left(s t^{(1)}, s t^{(2)}, \cdots s t^{(A)}\right)$.

for move $=1: K,\{$

a) The $i^{\text {th }}$ ant selects $\underline{\underline{b}}_{s u}$ element values with probability

$$
\begin{aligned}
p^{(i)}(\text { move })= & P T\left(2,\left(s t^{(i)}+\operatorname{move}\right) \bmod K\right) \\
& \times D\left(\left(s t^{(i)}+\text { move }\right) \bmod K\right) \\
& \forall i=1,2, \cdots A
\end{aligned}
$$

This probability is evaluated for for all ants.

b) Store the selected elements in $\underline{b}^{(i)} \forall i=1 \cdots N$.

c) The 2-dimensional indices of chosen locations in $P T$ constitute the trail for each ant. Store the trail for the $i^{t h}$ ant in $\operatorname{Tr}^{(i)} \in \mathrm{I}^{2 \times K} \forall i=$ $1 \cdots N$.

- The $1^{\text {st }}$ row \& $2^{\text {nd }}$ row of $T r^{(i)}$ represents row and column indexes of selected PT locations, respectively.

\}

2) if $Q\left(\underline{b}^{(i)}\right) \geq S_{E} \cdot Q\left(\underline{b}_{\text {elite }}\right)$ [Check for "elite" ants (note: $S_{E}$ is a scale parameter denoting the threshold value for elite ants)]

- Deposit pheromones:

$$
\begin{gathered}
P T\left(\operatorname{Tr}^{i}(1, k), \operatorname{Tr}(2, k)\right) \quad= \\
P T(\operatorname{Tr}(1, k), \operatorname{Tr}(2, k))+\triangle P T \quad,
\end{gathered}
$$

where $\triangle P T=D R \times \frac{f\left(\underline{b}^{(i)}\right)}{K}$

3) if $Q\left(\underline{b}^{(i)}\right) \leq S_{W} \cdot Q\left(\underline{b}_{\text {elite }}\right)$ [Check for "weak" ants (note: $S_{W}$ is a scale parameter denoting the threshold value for weak ants)]

- Evaporate pheromones:

$$
\begin{gathered}
P T\left(\operatorname{Tr}^{i}(1, k), \operatorname{Tr}(2, k)\right) \\
P T(\operatorname{Tr}(1, k), \operatorname{Tr}(2, k))-\triangle P T
\end{gathered}=
$$

4) Evaporate pheromones:

$$
P T(m, n)=P T(m, n) \times(1-E R) \forall m, n
$$

5) if $Q\left(\underline{b}^{(i)}\right)>Q\left(\underline{b}_{\text {elite }}\right)$ [Check if new solution is the elitist solution]

$$
\underline{b}_{\text {elite }}=\underline{b}^{(i)}
$$

\}

- The final solution vector $\widehat{\underline{b}}_{A C O}$ =
argmax $\left\{Q\left(\underline{b}_{\text {elite }}\right), Q\left(\underline{b}_{p h}\right)\right\}$
where $\underline{b}_{p h}$ is the trail
with the highest pheromone concentration. In order to compare complexity of the ACO based MUD with the optimal MUD, we define the product $\Upsilon=A \times V$ as the order of the computational complexity of our algorithm (e.g., an ACO with 8 ants and 100 iterations result in a $\Upsilon=800$ ).

\section{Performance Results}

We evaluate the ACO based MUD performance for a synchronous uplink MC-CDMA system uplink with: (1) $N=16$ carriers; (2) $K=16$ users; (3) Hadamard Walsh spreading codes; (4) BPSK modulation, and (5) four-fold frequency diversity. The following ACO parameters were employed: number of ants, $A=8 ; V=25(\Upsilon=200)$ and $V=100$ $(\Upsilon=800)$. Since the order of the complexity of this optimal MUD (employing BPSK modulation) is $2^{16}=65636$, the savings in complexity for $V=25$ and $V=100$ are $99.7 \%$ and $98.7 \%$, respectively. 


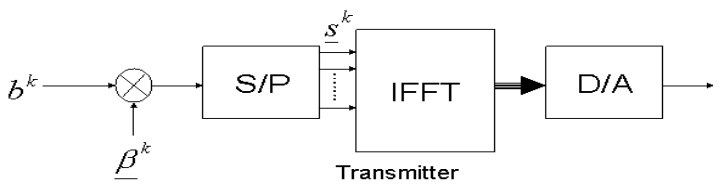

(a)

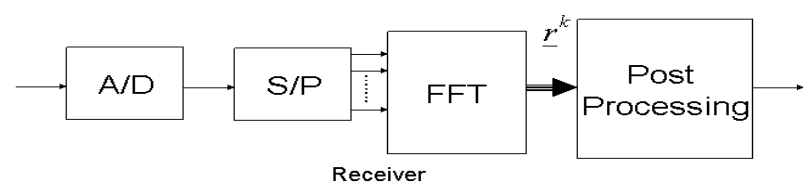

(b)

Fig. 1. $k^{\text {th }}$ user transmitter and receiver block diagram

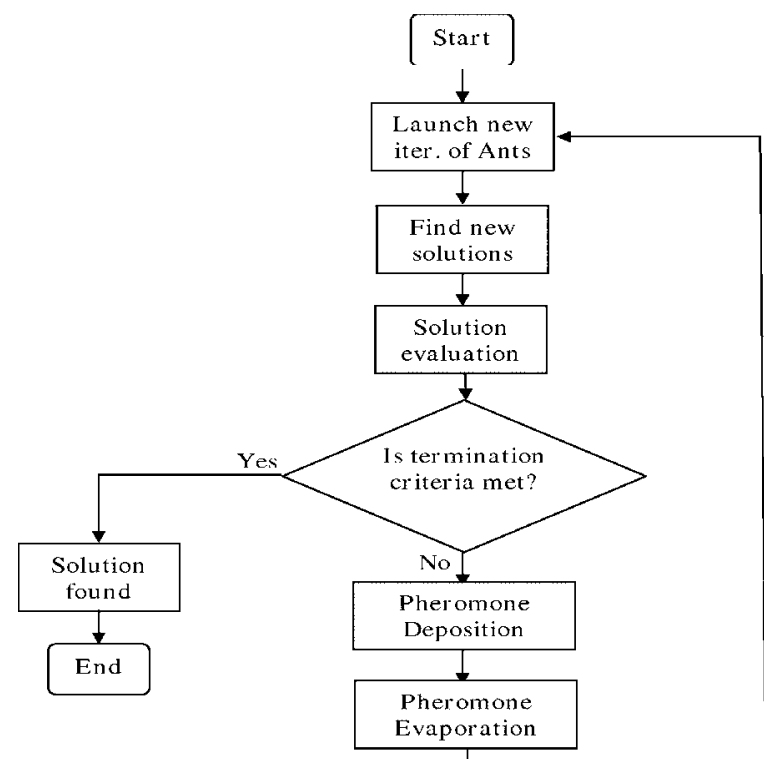

Fig. 2. A flowchart depicting the structure of ACO algorithm

Figure 3 presents four signal to noise ratio (SNR) vs. BER curves. The top most curve represents the maximum ratio combining (MRC) receiver BER performance. The remaining three curves show the performance of the optimal MUD and the performance of the ACO based MUD with $V=25$ and $V=100$. From Figure 3, it is evident that the MRC-based single user receiver has the worst performance. Furthermore, the ACO based MUDs approach the performance of the optimal MUD. Specifically, the ACO based MUD with $V=100$ matches the performance of the optimal MUD. Moreover, it is possible to decrease the ACO complexity to $V=25$ (by a factor of four versus the $V=100$ case) and only suffer a $1 \mathrm{~dB}$ loss in performance at a BER of $4 \cdot 10^{-3}$. While the ACO approach significantly outperforms an exhaustive search technique, it is important to remember that the ACO based MUD requires additional memory to store pheromone table.

\section{Conclusions}

This paper presents a novel low complexity algorithm that employs ACO to implement an optimal MUD for MC-CDMA synchronous up-links. To the best of authors' knowledge, this is the first attempt to apply swarm intelligence to MUD

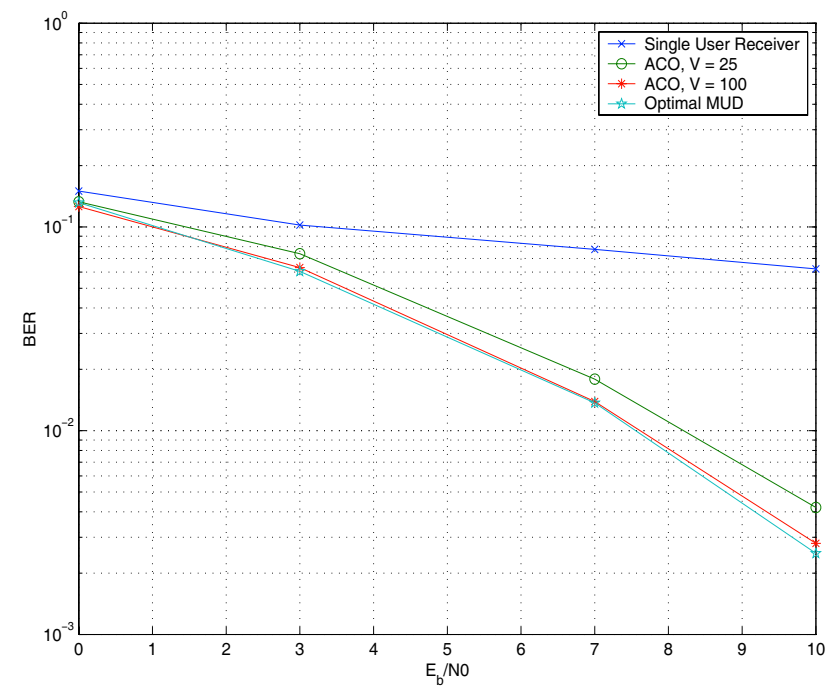

Fig. 3. Performance of Single User Receiver, Optimal MUD, and ACO-based MUD

design. Our ACO-based MUD matches the BER performance of the optimal MUD with more than $98 \%$ savings in terms of computational complexity. Moreover, we demonstrate that we can decrease the number of iterations in the ACO by a factor of four and only suffer a $1 \mathrm{~dB}$ performance loss relative to the optimal MUD.

\section{REFERENCES}

[1] S. Hara and R. Prasad, "Overview of multicarrier CDMA," IEEE Communications Magazine, vol. 35, pp. 126 - 133, December 1997.

[2] A. J. Viterbi, CDMA : Principles of Spread Spectrum Communication (Addison-Wesley Wireless Communications). Upper Saddle River, NJ: Prentice Hall PTR, 1995.

[3] L. L. Chong and L. B. Milstein, "Comparing DS-CDMA and multicarrier CDMA with imperfect channel estimation," in IEEE Signal Processing Workshop on Statistical Signal Processing, pp. 385 - 388, August 2001.

[4] S. Verdu, MultiUser Detection. Cambridge University Press, 1 ed., 1998.

[5] L. Brunel, "Multiuser detection techniques using maximum likelihood sphere decoding in multicarrier CDMA systems," IEEE Transactions on Wireless Communications, vol. 3, pp. 949 - 957, May 2004.

[6] C. Ibars and Y. Bar-Ness, "Comparing the performance of coded multiuser OFDM and coded MC-CDMA over fading channels," in Proceedings from the IEEE Global Telecommunications Conference, vol. 2, pp. 25-29, 2001.

[7] T. Fogarty, "Using the genetic algorithm to adapt intelligent systems," IEE Colloquium onjsac Symbols Versus Neurons, vol. 12, pp. 4/1-4/4, Oct. 1990.

[8] D. Fogel, "What is evolutionary computation?," IEEE Spectrum, vol. 37, pp. 28-32, Feb. 2000

[9] J. Kennedy and R. Eberhart, "Particle swarm optimization," IEEE International Conference on Neural Networks, vol. 4, pp. 1942-1948, Dec. 1995.

[10] M. Dorigo, L. Gambardella, M. Middendorf, , and T. Stutzle, "Guest editorial: special section on ant colony optimization," Aug. 2002.

[11] M. Dorigo and L. Gambardella, "Ant colony system: a cooperative learning approach to the traveling salesman problem," IEEE Transactions on Evolutionary Computation, vol. 1, pp. 53 - 66, Apr. 1997.

[12] S. L. Hijazi and B. Natarajan, "Novel low-complexity DS-CDMA multiuser detector based on ant colony optimization." Submitted for publication to IEEE Vehicular Technology Conference, 2004.

[13] W. C. Jakes, Microwave Mobile Communications. New York: IEEE Press, 1974

[14] W.Xu and L.B.Milstein, "Performance of multicarrier DS CDMA systems in the pres-ence of correlated fading," IEEE 47th Vehicular Technology Conf., vol. 3, pp. 2050-2054, May 1997. 